Cochrane Database of Systematic Reviews

\title{
Mass drug administration for filariasis: community views and programme design influences - a qualitative evidence synthesis (Protocol)
}

Taylor M, Oliver S, Garner P

Taylor M, Oliver S, Garner P.

Mass drug administration for filariasis: community views and programme design influences - a qualitative evidence synthesis (Protocol).

Cochrane Database of Systematic Reviews 2020, Issue 6. Art. No.: CD013638.

DOI: 10.1002/14651858.CD013638.

www.cochranelibrary.com

Mass drug administration for filariasis: community views and programme design influences - a qualitative evidence synthesis (Protocol) 
TABLE OF CONTENTS

HEADER 1

ABSTRACT

BACKGROUND

Figure 1.

OBJECTIVES

METHODS

ACKNOWLEDGEMENTS

REFERENCES

ADDITIONAL TABLES

APPENDICES

HISTORY

CONTRIBUTIONS OF AUTHORS

DECLARATIONS OF INTEREST

SOURCES OF SUPPORT 
[Qualitative Protocol]

\section{Mass drug administration for filariasis: community views and programme design influences - a qualitative evidence synthesis}

Melissa Taylor ${ }^{1}$, Sandy Oliver 2 , Paul Garner ${ }^{1}$

1Department of Clinical Sciences, Liverpool School of Tropical Medicine, Liverpool, UK. 2EPPI-Centre, Social Science Research Unit, UCL Institute of Education, University College London, London, UK

Contact address: Melissa Taylor, melissa.taylor@lstmed.ac.uk.

Editorial group: Cochrane Infectious Diseases Group.

Publication status and date: New, published in Issue 6, 2020.

Citation: Taylor M, Oliver S, Garner P. Mass drug administration for filariasis: community views and programme design influences - a qualitative evidence synthesis (Protocol). Cochrane Database of Systematic Reviews 2020, Issue 6. Art. No.: CD013638. DOI: 10.1002/14651858.CD013638.

Copyright (c) 2020 The Cochrane Collaboration. Published by John Wiley \& Sons, Ltd.

\section{A B S T R A C T}

\section{Objectives}

This is a protocol for a Cochrane Review (qualitative). The objectives are as follows:

- To synthesize qualitative research evidence about community experience with, and understanding and perception of, MDA programmes for filariasis

- To explore whether programme design and delivery influence the community experience identified in the analysis

We have intentionally kept the objectives broad, and we may modify them as we learn more from our analysis of the included studies, as is appropriate in qualitative research. 


\section{B A C K G R O U N D}

\section{Description of the topic}

Lymphatic filariasis (LF) is one of the neglected tropical diseases (NTDs), which are communicable diseases endemic to tropical and subtropical countries that cause considerable ill health, particularly among the poor (Molyneux 2013). The World Health Organization (WHO) recommends mass drug administration (MDA) to control all five of the most common NTDs: LF, onchocerciasis, soiltransmitted helminths, schistosomiasis, and trachoma (Webster 2014). Symptomatic chronic filarial infection causes limb and genital swelling, peeling of the skin, and fevers. Without treatment, the infection persists, and without early treatment, some of the effects are irreversible and can cause substantive morbidity and disability in adults who are infected (WHO 2019).

LF occurs in clearly geographically defined areas of the tropics and is transmitted by a variety of mosquito species, mainly species of the genus Anopheles or Culex. Repeated exposure to the bite of infected mosquitoes is required to infect people; hence vector control is an important preventive measure (WHO 2019). A variety of highly effective drugs are available for treatment, including diethylcarbamazine (DEC), ivermectin, and albendazole (WHO 2019), although debate surrounds the size of the effects seen with albendazole (Macfarlane 2019a).

Apart from vector control, the main tool for eliminating transmission of the disease is treatment of the disease through suppression of microfilaraemia with drugs known to be effective as tablets, or sometimes with medicated salt (Adinarayanan 2007). This suppression needs to be done for the whole population over long periods of time, and MDA to treat the whole population every year is the approach used most often (WHO 2019). In 2018, 893 million people required MDA to stop the spread of infection (WHO 2019).

The 2000 WHO global programme to eliminate lymphatic filariasis (GPELF) has been important and is ongoing; in addition, more integrative attempts are being made to treat multiple diseases through MDA programmes in which several drugs are given together to combat LF, soil-transmitted helminths, schistosomiasis, onchocerciasis, and trachoma (Webster 2014). Although it is clear that MDA if given properly really does help to reduce transmission, as anticipated some difficulties are involved in delivering at a local level (Parker 2013). Some researchers believe the large financial inputs (valued at USD 2 billion and USD 3 billion annually - Webster 2014) may inadvertently create pressure to highlight programme success without acknowledging these difficulties (Parker 2013).

Benefit in controlling these diseases is important because they afflict already poor and disadvantaged groups (Molyneux 2013); the size of the control programmes is considerable. By 2015, governments and donors had distributed over 6.7 billion tablets for treatment of LF alone (Specht 2019). However, to deliver medication on such a scale is a huge logistical task involving coordination between multiple sectors (Gyapong 2018). At the local level, village volunteers conduct house-to-house visits to identify, record, and inform the eligible population; this is followed by fixed day distribution (Allen 2011). Volunteers visit villages over a large geographical distance after completing a normal day's work. The work is unpaid and may have to be repeated if people were not at home for the first planned visit (Allen 2011). This can lead to shortcuts, with some distributors opting to give the tablets to one family member without ensuring that the entire family ingest them (Babu 2014). Finally, regular delivery of medicines assumes that people adhere to treatment. Although providers have the intention of doing public good, this does not always mean that communities are willing to take the drugs (Allen 2011). Problems with adherence have been reported in the literature, particularly regarding adverse effects (AEs) (Cabral 2017). Among people with active infection, AEs are common with treatment. The death of filarial parasites can cause a local inflammatory response; so when filarial load is high or worms are killed too rapidly, drug administration often leads to AEs (Kafle 2011). Mild reactions include fever and nausea, and serious AEs are those that lead to life-threatening or incapacitating conditions and hospitalization (Lima 2012). With MDA, it is not clear whether these AEs are common, as reporting and reporting accuracy are variable (Lima 2012; Wamae 2011; Weerasooriya 1998; WHO 2003). Although the uninfected population does not experience AEs, those who are infected may, and this could interfere with subsequent adherence to drug treatment, although these may not be important barriers to programmes providing MDA for LF (Mishra 2019)

\section{How the intervention might work}

In terms of drug effects, LF is treated with a two-drug combination consisting of albendazole and diethylcarbamazine, albendazole and ivermectin, or ivermectin and diethylcarbamazine. These treatments reduce microfilarial levels in the infected individual, and in some cases completely clear infection (Ismail 2001). When given to whole populations repeatedly over several years, MDA can reduce filaria levels to the point where transmission can no longer be sustained (Gyapong 2018). For this reason, the WHO currently recommends that at least $65 \%$ of the population should receive MDA for at least five years to achieve elimination (WHO 2019). When applying this strategy, 14 countries have been declared free of LF (Fang 2019).

In terms of delivery of the drug, MDA itself simply means giving the drug to whole populations, but this is a general term for a process that can take a variously organized and managed approach, with the various approaches themselves likely to influence the effectiveness of delivery. For example, MDA can be delivered by government health staff or by community health workers or through schools; it can be provided through mobile camps or by door-to-door visiting; the procedure may include careful household mapping and adherence recording, or very little attempt may be made to monitor actual ingestion.

Delivery may take place alongside health education or sensitization initiatives such as media campaigns or public activities, whereby people are given information on the purpose of MDA along with possible opportunities to raise concerns or queries. In addition, the whole process of planning MDA can vary from imposed programmes to full "collaborative" projects between external agencies and the community (Table 1). These programmes can be organised into top-down, bottom-up, and collaborative approaches, whereby the latter two have equal or majority contribution and governance from the communities intended to benefit (Whitehead 2002). Community involvement in planning and implementing interventions is thought to generate respect, trust, and sustainable support for the programme (Leise 2010), thereby facilitating community participation and engagement (Annamalai 2016). On the other hand, some point out that top- 
down approaches may be met with limited participation and missed opportunities to respond to problems arising at the local level (Silumbwe 2019; Sturmberg 2017), but these approaches may be simpler and more practical on a large scale. A recent analysis of top-down and bottom-up approaches to water, electricity, and sanitation initiatives suggests that in areas with weak economies, governance, and existing infrastructure, bottom-up approaches may be less effective and may require continued support from external actors (Annamalai 2016).

The way programmes are designed and delivered is likely to influence how people experience and perceive MDA. Therefore, in this review, we will carefully describe MDA programmes from all available data provided by the qualitative studies included, and in the analysis, we will explore how design influences community responses. We had considered exploring this relationship through qualitative comparative analysis, whereby quantitative and qualitative data are combined in a matrix to explore their relationship (Candy 2011). However, we intend to simply synthesize what people think of MDA and not necessarily identify how acceptability or compliance may be influenced. In addition, quantitative reviews of effectiveness that would be suitable to compare with this study are insufficient.

\section{How this review might inform or supplement what is already known in this area}

The Cochrane Database of Systematic Reviews includes two reviews regarding specific treatment regimens of MDA for LF. The first - Macfarlane 2019a - investigates the efficacy of albendazole, given alone or in combination; and the second - Adinarayanan 2007 - investigates the efficacy of DEC salt. This Cochrane Review will build on this by exploring how people experience these programmes but is not specifically linked to either of these Cochrane Reviews (Adinarayanan 2007; Macfarlane 2019a).

Before starting this review, we sought to examine the questions addressed, the studies included, and the conclusions drawn from similar reviews that explored community views and responses to LF mass treatment programmes. We identified three systematic reviews and one rapid review that synthesized evidence regarding delivery and adherence (Table 2). Two reviews collated evidence globally - one from India and another from sub-Saharan Africa. Data were collected from studies published up until 2012 (Krentel 2013), 2013 (Babu 2014), 2016 (Silumbwe 2017), or 2017 (Ames 2019). One quantitative and two mixed-methods studies largely based their findings on quantitative study designs, such as standardized surveys, which are poorly designed to capture two types of information: the first, unexpected or novel information as responses are predetermined; and the second, deeper levels of reasoning that highlight important beliefs or experiences that fuel such assertions. Boyd 2010 documents the challenges encountered by researchers when using the quantitative survey method; the response they most frequently received was 'don't know', and trial authors questioned whether this was proxy for 'don't care', or if another factor was present that was not being captured by the research. One mixed-methods study - Krentel 2013 supplemented study findings with informal interviews with five senior LF scientists, although the relationships of these scientists to MDA programmes and their risk of bias were not disclosed. This generated some interesting observations on behaviour at the ground level. A final article - a rapid review commissioned by the WHO (Ames 2019) - was conducted in part by researchers who have produced pieces of research advocating for MDA programmes (King 2011). Due to the nature of rapid reviews, limited time may have been provided to develop theory from the themes identified.

Facilitators outlined in the above studies include training (Ames 2019; Silumbwe 2017), community awareness (Silumbwe 2017), management of adverse effects (Ames 2019; Krentel 2013; Silumbwe 2017), trust (Ames 2019; Krentel 2013), community involvement in planning and conducting the MDA (Ames 2019), and whether timing was convenient (Ames 2019). Barriers include fear of adverse effects (Babu 2014; Krentel 2013), lack of perception of benefit (Babu 2014; Krentel 2013), lack of information or understanding (Ames 2019; Babu 2014; Krentel 2013), delays in drug delivery (Krentel 2013; Silumbwe 2017), lack of motivation of distributors (Krentel 2013), and inadequate numbers of distributors (Ames 2019). Silumbwe 2017 noted some interesting contextual factors influencing compliance, including the belief that MDA transmits Ebola, and the thought that MDA is not a priority during other outbreaks. This may suggest that it is useful to consider findings in terms of country and context.

The conceptual model was developed in light of the findings of these reviews and represents current understanding of the factors that influence adherence at the community level (Figure 1). The diagram aims to help us delineate our thinking before conducting the review, reading previous reviews, and exploring our own thoughts. The conceptual model is a guide that is likely to be revised in light of the findings of this review. 
Figure 1. Conceptual model of the driving and restraining influences on MDA delivery and adherence.

\section{Restraining forces}

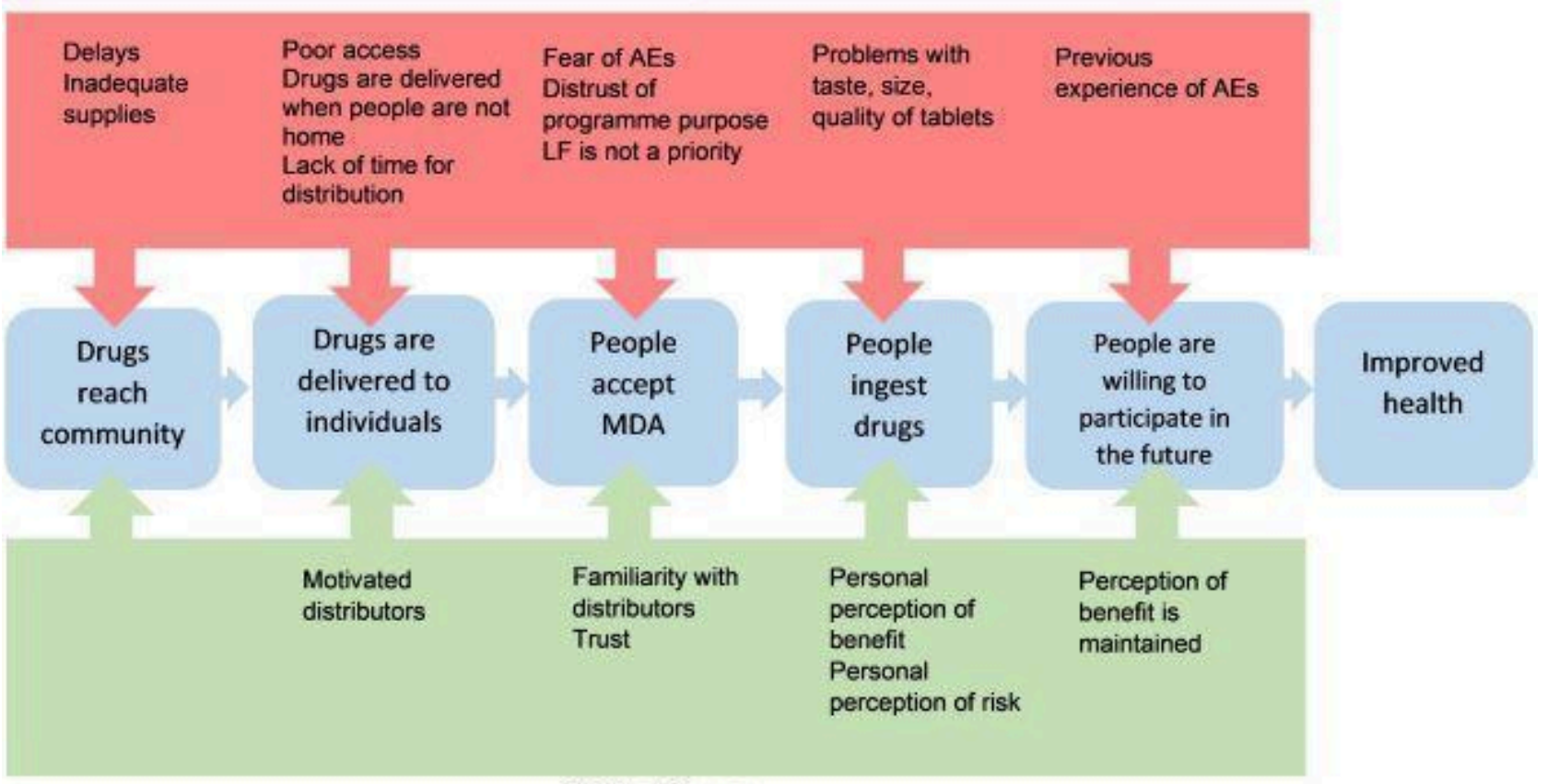

\section{Driving forces}

\section{Why is it important to do this review?}

Cochrane Reviews conducted previously have helped by listing barriers to and facilitators of adherence. Decisions to adhere to treatment are complicated, however, and often are related to multiple competing beliefs and values and the wider socio-political context. Therefore, we plan to conduct a careful analysis using recent methods in quality evidence synthesis (QES) (1) to further examine the context in which these drugs are delivered in an effort to explain when sometimes adherence is not as high, as is desired by policy-makers, and (2) to delineate community views to provide feedback on programme design. This approach provides the underpinning for this review, in which we aim to synthesize the perceptions and experiences of those receiving MDA with the goal of attaining a broader understanding of the impact of programmes and consumers' openness towards them.

\section{OB JECTIVES}

- To synthesize qualitative research evidence about community experience with, and understanding and perception of, MDA programmes for filariasis

- To explore whether programme design and delivery influence the community experience identified in the analysis

We have intentionally kept the objectives broad, and we may modify them as we learn more from our analysis of the included studies, as is appropriate in qualitative research.

\section{METHODS}

\section{Criteria for considering studies for this review \\ Types of studies}

This review will synthesize qualitative research including ethnographies, phenomenologies, qualitative process evaluations, and case studies. We define qualitative research as studies that collect data from ethnographic observations, in-depth interviews, focus group discussions, and open-ended survey questions. Appropriate analysis methods for qualitative research include thematic analysis, narrative analysis, framework analysis, and grounded theory (Thomas 2008).

We will include mixed-methods studies when it is possible to extract qualitative data.

We will not include studies that include qualitative data collection methods but report and analyse all data quantitatively.

We will restrict included studies to those from 2000 to present day, as this marks the date of the introduction of the Global Programme for the Elimination of Lymphatic Filariasis and the date that MDA activities were introduced and scaled up for filariasis (WHO 2019).

We will include both published and unpublished studies.

\section{Topics of interest}

- Phenomenon of interest: this review will consider community experiences, perceptions, or attitudes towards MDA programmes for LF

- Setting: we will include any setting in which MDA for filariasis was provided, as the purpose of synthesis to inform decision- 
making is to yield theories that are more transferable and socially relevant over a broader range of contexts

- Perspectives: we will include the perspectives of any community member eligible for the MDA programme and village leaders. Perceptions of lay healthcare workers (those without formal training or qualifications, including community health workers and drug distributors as defined by Lewin 2010) and government health workers may be included to triangulate results but will be clearly separated from the perspectives of the general consumer population. The literature on MDA policies and their implementation is extensive. This review is not concerned with understanding of policies by those implementing them; we do not therefore intend to summarize the views of those affiliated with the programme design or with programme governance

- Intervention: we will examine delivery of MDA, which, for this review, is defined as administration of an antifilarial drug to the entire at-risk population (irrespective of symptoms or infection) on a regular, often annual, basis. During scoping, it was identified that most of the available research related to compliance in MDA programmes focused on LF specifically. Therefore, this review will focus on community perceptions towards MDA programmes for LF

\section{Search methods for identification of studies}

We developed the search strategy in consultation with Cochrane Effective Practice and Organisation of Care (EPOC) Information Specialists.

\section{Electronic searches}

We will attempt to identify all relevant studies regardless of publication status (i.e. published, unpublished, in press, or in progress). We will search the following databases using the search terms and strategy described in Table 3 - Cochrane Infectious Diseases Group Specialized Register; MEDLINE (PubMed); Embase (OVID); Cochrane Central Register of Controlled Trials (CENTRAL), published in the Cochrane Library; Latin American and Caribbean Health Sciences Literature (LILACS (BIREME)); Cumulative Index to Nursing and Allied Health Literature (CINAHL (EBSCOHost)); Global Health; CAB Direct; and Science Citation Index - Expanded (all in the Web of Science). We will limit our searches from publication year 2000 onwards and to studies conducted in English.

We will also search the WHO International Clinical Trials Registry Platform (www.who.int/ictrp/search/), along with ClinicalTrials.gov, to identify ongoing trials, using the terms 'filariasis' and 'mass drug administration'.

\section{Searching other resources}

The Cochrane Qualitative and Implementation Methods Group recommends supplementary searching activities due to the limited availability of qualitative research. To achieve this, we will scan reference lists and perform citation searching of included studies and existing reviews identified in the Background. We will contact experts in the field to ask what they know about published and unpublished data.

\section{Grey literature}

We will search OpenGrey to identify grey literature (www.opengrey.eu).

\section{Selection of studies}

We will import all search results into EndNote, and will remove any duplicates. Two review authors (MT and Rebecca Thomas (RT)) will independently screen retrieved search results against the inclusion criteria. This two-step process will consist of screening first titles/abstracts, then full text. Using two review authors to screen is valuable in providing opportunities to explore relevance and meaning of study findings rather than to reach consensus (Soilemezi 2018). We will summarize this process in a PRISMA flow diagram detailing the numbers of studies removed and kept at each step. We will note the reasons for exclusion of full-text studies and will present this information in the supplementary material.

\section{Language translation}

Due to time and resource demands of translation, only studies available in English will be eligible for inclusion.

\section{Sampling of studies}

The previous version of this review - an unpublished masters dissertation (Taylor 2019) - returned only 14 studies, and a subsequent scoping search has not returned any additional studies. Therefore, we are not expecting to require a sampling strategy.

\section{Data extraction and management}

One review author (MT) will independently extract study data using a predefined data extraction form, which will include information such as study author, country, study aim, study participants, study methods, qualitative data collection methods, key study findings, conceptual models used, programme organization, community engagement, drug regimen, and rounds of MDA received at the time of the study.

\section{Assessing the methodological limitations of included studies}

We will assess quality by using a standardized set of criteria to impart some objective distance and to ensure consistency. We chose a modified version of the tool developed by the EPPI-centre for its clear and straightforward approach and use in a similar QES investigating consumer perceptions and experiences of a health intervention (Appendix 1; Eshun-Wilson 2019). This tool assessed the following criteria: rigour in sampling, rigour in data collection, rigour in analysis, grounding of data, and breadth and depth of study findings. Each criterion offered several prompts to aid the user in making a judgement. For each category, studies received a score of (1) Yes, a fairly thorough attempt was made, (2) Yes, several steps were taken, (3) Yes, a few steps were taken, or (4) No, not at all/not stated/can't tell. Two review authors (MT and RT) will independently conduct a quality assessment of each paper before comparing findings. We will discuss disagreements, and if consensus cannot be reached, we will consult a third review author.

We will not exclude studies based on our assessment of methodological limitations. However, we will use information about methodological limitations to assess our confidence in the review findings.

\section{Data management, analysis, and synthesis}

We will use thematic synthesis as described in Thomas 2008 and informed by the Braun 2006 thematic analysis. Thematic synthesis 
assumes that knowledge of reality is mediated by perceptions and beliefs, thus making it an appropriate choice for a study investigating perceptions, experiences, and acceptability of a health intervention. This method also assumes that findings are reproducible and transferable, which aligns with the second study objective - to explore implications for programme delivery. The steps to be taken are outlined below.

- Familiarising yourself with the data: review authors (MT and RT) will read through relevant background literature and the full length of studies included in the review to become familiar with and immersed in the data, noting any initial thoughts.

- Generating initial codes: working independently, review authors (MT and RT) will begin substantive coding, by determining which segments of text will be assigned simple codes that reflect their contents rather than researchers' preconceptions. This will include both first- and second-order data, with firstorder data being the original quotations, and second order data comprising study authors' interpretations. Efforts will be made to retain accounts that differ from the emerging understanding of the situation. Review authors will then compare individual codes on a study-by-study basis to reach consensus on the appropriateness and terminology of each code. The result of this process will be the development of a shared coding framework that can be applied to subsequent papers, which may be refined and amended as new codes emerge.

\section{Description of delivery}

In addition, we will attempt to code for aspects of the delivery system that may have contributed to the emerging perceptions and acceptability of those receiving MDA. These will include:

- who delivers: community health workers, government health staff, or teachers;

- how delivered: through schools, through mobile camps, by door-to-door visiting, by using household mapping;

- adherence monitoring: systems of adherence recording in place or no such systems; and

- health education and sensitization: with or without concurrent health education or sensitization initiatives such as media campaigns or public activities.

\section{Community engagement}

We will try to categorize using the seven delivery methods outlined in Whitehead 2002, or at least by grouping these into three categories: top-down, bottom-up, and collaborative. When this information is not available from the report, we will seek other documents related to MDA policies in the country at the time to try to input some basic characteristics of the programme, and we will note that the characteristics are secondarily derived from other sources. To find this information, we first will screen citations of the target study, then will refer to policy documents including Macfarlane 2019b, and finally will perform a Google search for other documents that refer to these programmes.

\section{Thematic methods}

- Searching for themes: working together, we will group codes into potential themes, gathering all data relevant to each theme. Here, review authors will interpret meaning behind the data and will think about the relationships between codes, themes, and hierarchies of themes. MT, RT, and PG will hold regular meetings to reflect on emerging themes.

- Reviewing themes: review authors will ensure that the pattern of data within themes is coherent, and that there is a clear distinction between themes and subthemes. This may involve breaking, merging, or removing themes with too little, too much, or disparate information. We will review included studies a second time to capture any data missed for newly emerging themes.

- Defining and naming themes: we will refine the overall story and terminology of each theme.

\section{Team roles}

\section{Producing the report}

One review author (MT) will produce a narrative of findings for each theme, integrating illustrative quotes that are vivid and lack unnecessary complexity. We will discuss findings in relation to the original research question and how they fit within the conceptual model developed in the Background section. We will analyse and discuss findings specific to any geographical settings or contexts, such as settings also receiving MDA for other co-endemic diseases, poverty levels, and programme design (such as form of delivery, drug regimen, and rounds of MDA received at the time of the study), as well as differences in perceptions between male and female community members.

Finally, we will update the conceptual model to reflect the new understanding, and we will discuss any developments.

We will use Atlas.ti software to manage the data.

\section{Assessing our confidence in review findings}

Two review authors (MT and RT) will use the GRADE-CERQual (Confidence in the Evidence from Reviews of Qualitative Research) approach to assess our confidence in each finding (Lewin 2018). CERQual assesses confidence in the evidence based on the following four key components.

- Methodological limitations of included studies: the extent to which there are concerns about the design or conduct of the primary studies that contributed evidence to an individual review finding.

- Coherence of the review finding: how clear and cogent the fit is between data from the primary studies and a review finding that synthesizes those data. By 'cogent', we mean well supported or compelling.

- Adequacy of the data contributing to a review finding: the degree of richness and the quantity of data supporting a review finding.

- Relevance of included studies to the review question: how the body of evidence from the primary studies supports a review finding. This information is applicable to the context (perspective or population, phenomenon of interest, setting) specified in the review question.

After assessing each of the four components, we will make a judgement about our overall confidence in the evidence supporting the review finding. We will judge confidence as high, moderate, low, or very low. We will base the final assessment on consensus among the review authors. All findings will start as high confidence and 
then will be downgraded if there are important concerns regarding any of the CERQual components.

\section{Sensitivity analysis}

As we intend to include all studies in the synthesis, regardless of quality assessment, we will conduct a sensitivity analysis to ascertain how removing studies assessed as 'low quality' will affect content and confidence in findings.

\section{'Summary of qualitative findings' table and evidence profile}

We will present summaries of findings and our assessments of confidence in these findings in the 'Summary of qualitative findings' table. We will present detailed descriptions of our confidence assessment in an evidence profile.

\section{Review author reflexivity}

In qualitative research, we appreciate that the background and position of researchers will shape interpretation of results, and thus team positionality at the outset, through the process of analysis and synthesis. We state these broad positionality statements at the outset here. PG was the clinician organizing the delivery of MDA for LF as part of a research project in the Maprik Area of the West Sepik in the 1980s. These populations were heavily diagnosed with filariasis, and the DEC made people unwell, so he has seen adverse effects first-hand. As a public health professional, his values and principles include believing that health professionals take account of views of the public on clinical and public health policies. His research reflects these values. MT, RT, and SO have no personal experience regarding MDA programmes and hold differing perspectives on their value. RT is working on a project on human rights and guideline development and is sensitive to policies from a human rights perspective. SO is ambivalent about MDA programmes and views them from the standpoint of families rather than practitioners. The work will build on MT's thesis, which highlighted several consumer concerns about the programme. Before she conducted this research, MT's views on
MDA were influenced by her academic tuition to date, which involves a provider-centred rhetoric that MDA is a highly effective and appropriate solution.

Analysis will be conducted by two primary analysts (MT and RT), who will additionally provide feedback on their findings and interpretations to the whole research team. This will involve regular meetings with PG and occasional meetings with SO. As different researchers will approach the analysis from different perspectives, this collaborative effort should produce a richer, more nuanced understanding of a complex situation while generating opportunities to identify and contest any assumptions or beliefs held by individual review authors. To further increase reflexivity in our research design, we will aim to explore and explain any findings that appear to contradict our understanding of the situation. Primary analysts will keep memo notes during the initial stages of analysis to provide a transparent account of the interpretation process and the development of themes.

\section{ACKN OWLEDGEMEN TS}

The Academic Editor is Dr Ingrid Eshun-Wilson. We are grateful to Dr Hellen Gelband for her input to this protocol also, and to Signoff Editor Professor Lisa Bero.

The editorial base of the Cochrane Infectious Diseases Group (CIDG) is funded by aid from the UK government for the benefit of low- and middle-income countries (project number 300342-104).

Melissa Taylor, Sandy Oliver, and Paul Garner are supported by the Research, Evidence and Development Initiative (READ-It) project. READ-It (project number 300342-104) is funded by UK aid from the UK government; however, the views expressed do not necessarily reflect the UK government's official policies.

We thank Rebecca Thomas (CIDG Research Associate), who will join the author team at review stage, for her input to this protocol.

We are grateful to peer reviewers Meike Zuske and Professor Ruth Garside for their constructive comments. 


\section{RE F E R E N C E S}

\section{Additional references}

\section{Adinarayanan 2007}

Adinarayanan S, Critchley JA, Das PK, Gelband H.

Diethylcarbamazine (DEC)-medicated salt for community-based control of lymphatic filariasis. Cochrane Database of Systematic Reviews 2007, Issue 1. [DOI: 10.1002/14651858.CD003758.pub2]

\section{Allen 2011}

Allen T, Parker M. The 'other diseases' of the millennium development goals: rhetoric and reality of free drug distribution to cure the poor's parasites. Third World Quarterly 2011; 32(1):91-117.

\section{Ames 2019}

Ames HMR, Zuske M, King JD, Steinmann P, Bosch-Capblanch X. Community and drug distributor perceptions and experiences of mass drug administration for the elimination of lymphatic filariasis: a rapid review of qualitative research. Advances in Parasitology 2019; 103:117-49.

\section{Annamalai 2016}

Annamalai TR, Devkar G, Mahalingam A, Benjamin S, Rajan SC, Deep A. What is the evidence on top-down and bottom-up approaches in improving access to water, sanitation and electricity services in low-income or informal settlements? November 2016. https://eppi.ioe.ac.uk/CMS/Portals/0/ PDF\%20reviews\%20and\%20summaries/SANITATION \%202016\%20Annamalai.pdf (accessed 2 April 2020).

\section{Babu 2014}

Babu BV, Babu GR. Coverage of, and compliance with, mass drug administration under the programme to eliminate lymphatic filariasis in India: a systematic review. Transactions of The Royal Society of Tropical Medicine and Hygiene 2014; 108(9):538-49.

\section{Boyd 2010}

Boyd A, Won KY, McClintock SK, Donovan CV, Laney SJ, Williams SA, et al. A community-based study of factors associated with continuing transmission of lymphatic filariasis in Leogane, Haiti. PLoS Neglected Tropical Diseases 2010; 4(3):e640.

\section{Braun 2006}

Braun V, Clarke V. Using thematic analysis in psychology. Qualitative Research in Psychology 2006; 3(2):77-101.

\section{Cabral 2017}

Cabral S, Bonfim C, Oliveira R, Oliveira P, Guimarães T, Brandão $\mathrm{E}$, et al. Knowledge, attitudes and perceptions regarding lymphatic filariasis: study on systematic noncompliance with mass drug administration. Revista do Instituto de Medicina Tropical de Sao Paulo 2017; 59:e23.

\section{Candy 2011}

Candy B, King M, Jones L, Oliver S. Using qualitative synthesis to explore heterogeneity of complex interventions. BMC Medical Research Methodology 2011; 11(124):1-9.

\section{Eshun-Wilson 2019}

Eshun-Wilson I, Rohwer A, Hendricks L, Oliver S, Garner P. Being HIV positive and staying on antiretroviral therapy in Africa: a qualitative systematic review and theoretical model. PLoS One 2019; 14(1):e0210408.

\section{Fang 2019}

Fang Y, Zhang Y. Lessons from lymphatic filariasis elimination and the challenges of post-elimination surveillance in China. BMC Infectious Diseases of Poverty 2019; 8:66.

\section{Gyapong 2018}

Gyapong JO, Owusu IO, da-Costa Vroom FB, Mensah EO, Gyapong M. Elimination of lymphatic filariasis: current perspectives on mass drug administration. Research and Reports in Tropical Medicine 2018; 9:25-33.

\section{Ismail 2001}

Ismail MM, Jayakody RL, Weil GJ, Deepika F, De Silva GAC, Balasooriya WK. Long-term efficacy of singledose combinations of albendazole, ivermectin and diethylcarbamazine for the treatment of bancroftian filariasis. Transactions of The Royal Society of Tropical Medicine and Hygiene 2001; 95(3):332-5.

\section{Kafle 2011}

Kafle K. Investigation of serious adverse events following mass drug administration (MDA) 2011 for lymphatic filariasis elimination in Nepal. 2011. www.researchgate.net/ publication/279195304_Investigation_of_serious_adverse_events_followin (accessed 15 December 2019).

\section{King 2011}

King JD, Zielinski-Gutierrez E, Pa'au M, Lammie P. Improving community participation to eliminate lymphatic filariasis in American Samoa. Acta Tropica 2011; 120:S48-54.

\section{Krentel 2013}

Krentel A, Fischer PU, Weil GJ. A review of factors that influence individual compliance with mass drug administration for elimination of lymphatic filariasis. PLoS Neglected Tropical Diseases 2013; 7(11):e2447.

\section{Leise 2010}

Liese B, Rosenberg M, Schratz A. Programmes, partnerships, and governance for elimination and control of neglected tropical diseases. Lancet 2010; 375(9708):67-76.

\section{Lewin 2010}

Lewin S, Munabi-Babigumira S, Glenton C, Daniels K, Bosch-Capblanch X, van Wyk BE, et al. Lay health workers in primary and community health care for maternal and child health and the management of infectious diseases. Cochrane Database of Systematic Reviews 2010, Issue 3. [DOI: 10.1002/14651858.CD004015.pub3]

\section{Lewin 2018}

Lewin S, Booth A, Glenton C, Munthe-Kaas H, Rashidian A, Wainwright $\mathrm{M}$, et al. Applying GRADE-CERQual to qualitative 
evidence synthesis findings: introduction to the series. Implementation Science 2018; 13:2.

\section{Lima 2012}

Lima AW, Medeiros Z, Santos ZC, Costa GM, Braga C. Adverse reactions following mass drug administration with diethylcarbamazine in lymphatic filariasis endemic areas in the Northeast of Brazil. Revista de Sociedade Brasileira de Medicina Tropical 2012; 45(6):745-50.

\section{Macfarlane 2019a}

Macfarlane C, Budhathoki SS, Johnson S, Richardson M, Garner P. Albendazole alone or in combination with microfilaricidal drugs for lymphatic filariasis. Cochrane Database of Systematic Reviews 2019, Issue 1. [DOI: 10.1002/14651858.CD003753.pub4]

\section{Macfarlane 2019b}

Macfarlane C, Dean L, Thomson R, Garner P. Community drug distributors for mass drug administration in neglected tropical disease programmes: systematic review and analysis of policy documents. Journal of Global Health 2019; 9(2):020414.

\section{Mishra 2019}

Mishra S. Over 1,300 fall ill at filariasis vaccination drive in Jharkhand; villagers attack health staff. www.hindustantimes.com/ranchi/over-1-300-fall-ill-at-filariasisvaccination-drive-in-jharkhand-villagers-attack-healthstaff/story-CCWxD4ztoh62gBFUxUVDWK.html (accessed 10 December 2019).

\section{Molyneux 2013}

Molyneux D. Neglected tropical diseases. Community Eye Health Journal 2013; 26(82):21-4

\section{Parker 2013}

Parker M, Allen T. Will mass drug administration eliminate lymphatic filariasis? Evidence from northern coastal Tanzania. Journal of Biosocial Science 2013; 45(4):517-45.

\section{Silumbwe 2017}

Silumbwe A, Zulu JM, Halwindi H, Jacobs C, Zgambo J, Dambe $R$, et al. A systematic review of factors that shape implementation of mass drug administration for lymphatic filariasis in sub-Saharan Africa. BMC Public Health 2017; 17(1):484.

\section{Silumbwe 2019}

Silumbwe A, Halwindi H, Zulu JM. How community engagement strategies shape participation in mass drug administration programmes for lymphatic filariasis: the case of Luangwa District, Zambia. PLOS Neglected Tropical Diseases 2019; 13(11):e0007861.

\section{Soilemezi 2018}

Soilemezi D, Linceviciute S. Synthesizing qualitative research: reflections and lessons learnt by two new reviewers. International Journal of Qualitative Methods 2018; 17:1-14.

\section{Specht 2019}

Specht S, Suma TK, Pedrique B, Hoerauf A. Elimination of lymphatic filariasis in South East Asia. BMJ 2019; 364:k5198.

\section{Sturmberg 2017}

Sturmberg JP, Njoroge A. People-centred health systems, a bottom-up approach: where theory meets empery. Journal of Evaluation in Clinical Practice 2017; 23:467-73.

\section{Taylor 2019}

Taylor M. Mass drug administration for lymphatic filariasis: qualitative evidence synthesis of community views [Master thesis]. Liverpool, UK: Liverpool School of Tropical Medicine, 2019.

\section{Thomas 2008}

Thomas J, Harden A. Methods for the thematic synthesis of qualitative research in systematic reviews. BMC Medical Research Methodology 2008; 8(45):1-10.

\section{Wamae 2011}

Wamae CN, Njenga SM, Ngugi BM, Mbui J, Njaanake HK. Evaluation of effectiveness of diethylcarbamazine/albendazole combination in reduction of Wuchereria bancrofti infection using multiple infection parameters. Acta Tropica 2011; 120:S33-8.

\section{Webster 2014}

Webster JP, Molyneux DH, Hotez PJ, Fenwick A. The contribution of mass drug administration to global health: past, present and future. Philosophical Transactions of The Royal Society $B$ Biological Sciences 2014; 369(1645):20130434.

\section{Weerasooriya 1998}

Weerasooriya MV, Kimura E, Dayaratna DA, Weerasooriya TR, Samarawickrema WA. Efficacy of a single dose treatment of Wuchereria bancrofti microfilaria carriers with diethylcarbamazine in Matara, Sri Lanka. Ceylon Medical Journal 1998; 43(3):151-5.

\section{Whitehead 2002}

Whitehead TI. Community based interventions, definitions and types. Cultural Ecology of Health and Change (CEHC) Working Papers Series 2002; 2:3-4.

\section{WHO 2003}

World Health Organization. Report on active surveillance for adverse events following the use of drug co-administrations in the Global Programme to Eliminate Lymphatic Filariasis. Weekly Epidemiological Report 2003; 78(36):313-20.

\section{WHO 2019}

World Health Organization. Lymphatic filariasis: Global Programme to Eliminate Lymphatic Filariasis. www.who.int/ lymphatic_filariasis/elimination-programme/en/ (accessed 15 December 2019).

\section{ADDITIONAL TABLES}


Table 1. Types of delivery in MDA programmes

\begin{tabular}{|c|c|c|}
\hline Form of delivery & Definition & Approach \\
\hline Type I & $\begin{array}{l}\text { "Programs in which individuals or groups/organizations indigenous to the community to } \\
\text { be served by a program (target community) initiate, without any external (to that com- } \\
\text { munity) support" }\end{array}$ & $\begin{array}{l}\text { Bottom-up } \\
\text { Bottom up }\end{array}$ \\
\hline Type II & $\begin{array}{l}\text { "Programs in which individuals or community groups/organizations groups/ organiza- } \\
\text { tions indigenous to the community initiate initiate, and recruit external external, techni- } \\
\text { cal (expertise) support" }\end{array}$ & $\begin{array}{l}\text { Bottom-up } \\
\text { Bottom up }\end{array}$ \\
\hline Type III & $\begin{array}{l}\text { "Programs in which individuals or community-based community based organizations } \\
\text { (CBOs) pursue external fiscal support or funding" }\end{array}$ & $\begin{array}{l}\text { Bottom-up } \\
\text { Bottom up }\end{array}$ \\
\hline Type IV & $\begin{array}{l}\text { "Programs in which individuals or CBOs indigenous to the target community initiate and } \\
\text { recruit external technical and fiscal support" }\end{array}$ & $\begin{array}{l}\text { Bottom-up } \\
\text { Bottom up }\end{array}$ \\
\hline Type V & $\begin{array}{l}\text { "Programs which are initiated by external change agencies (public or private organiza- } \\
\text { tion, university, a corporation, a foundation or some other philanthropic group, and so } \\
\text { on) within a target community, but [are done] does it without any input from individual } \\
\text { residents or organizations of that community, except as program recipients" }\end{array}$ & $\begin{array}{l}\text { Top-down } \\
\text { Top down }\end{array}$ \\
\hline Type VI & $\begin{array}{l}\text { "Programs which are planned and initiated by external change agencies, and community } \\
\text { members are eventually invited to participate on community advisory committees, or as } \\
\text { lower-level lower level project staff such as 'community "community outreach workers', } \\
\text { workers", or as volunteers" }\end{array}$ & $\begin{array}{l}\text { Top-down } \\
\text { Top down }\end{array}$ \\
\hline Type VII & $\begin{array}{l}\text { "Programs which are planned and implemented as an equitable partnership by CBOs and } \\
\text { an external change agent or technical organization" }\end{array}$ & Collaborative \\
\hline
\end{tabular}

Abbreviations: MDA: mass drug administration.

Amended from Whitehead 2002.

Table 2. Characteristics of review studies

\begin{tabular}{|c|c|c|c|c|}
\hline Author & Aims & Methods & Inclusion criteria & $\begin{array}{l}\text { Number } \\
\text { of studies }\end{array}$ \\
\hline Babu 2014 & $\begin{array}{l}\text { "To systematically review pub- } \\
\text { lished studies on the coverage } \\
\text { of and compliance with MDA } \\
\text { under the Programme for the } \\
\text { Elimination of Lymphatic Filar- } \\
\text { iasis (PELF) in India" }\end{array}$ & $\begin{array}{l}\text { Quantita- } \\
\text { tive }\end{array}$ & $\begin{array}{l}\text { - Up to } 2013 \\
\text { - India } \\
\text { - Quantitative studies and quantitative data that can be } \\
\text { extracted from mixed-methods studies } \\
\text { "Community based studies that evaluated MDA coverage } \\
\text { and compliance conducted by the health services. Data } \\
\text { were excluded if governmental, nongovernmental or re- } \\
\text { search organisations intervened to improve compliance. } \\
\text { However, papers that reported such studies were consid- } \\
\text { ered and data on control MDAs were included" }\end{array}$ & 36 \\
\hline $\begin{array}{l}\text { Silumbwe } \\
2017\end{array}$ & $\begin{array}{l}\text { "To systematically document } \\
\text { the barriers and facilitators to } \\
\text { implementation of MDA for LF } \\
\text { in Sub-Saharan Africa" }\end{array}$ & $\begin{array}{l}\text { Mixed- } \\
\text { methods: } \\
\text { Qualita- } \\
\text { tive }(n=3)\end{array}$ & $\begin{array}{l}\text { - } 2000 \text { to } 2016 \\
\text { - Sub-Saharan Africa } \\
\text { - Studies that assessed the following outcomes: “(i) treat- } \\
\text { ment coverage/compliance, (ii) program sustainability, }\end{array}$ & 18 \\
\hline
\end{tabular}


Table 2. Characteristics of review studies (Continued)

Quantita-

tive $(n=4)$

Mixed

methods

$(n=4)$

Pro-

gramme

reports ( $\mathrm{n}$

$=6$ )

Evalua-

tion $(\mathrm{n}=$

1) (iii) successful implementation referring to perceptions among implementation stakeholders (both provider and community) that a given treatment, service, practice, or innovation is agreeable, palatable, or satisfactory with their needs, and (iv) community participation, defined as the involvement of the community in programme design implementation and evaluation"
Krentel "To attempt to identify factors

2013 and patterns that are associated with compliance with MDA that apply across countries and cultures"
Mixed-

methods: proportion of qualitative and quantitative studies unclear
- 2000 to 2012

- Global

- "Studies that: (i) reviewed the literature on compliance with MDA for LF; (ii) described or assessed factors associated with compliance with MDA for LF; (iii) analysed, observed, or documented compliance rates with MDA and/ or provided an explanation or discussion of the rates; and (iv) were identified from reference lists of primary papers"

\begin{tabular}{llll}
\hline $\begin{array}{l}\text { Ames } \\
2019\end{array}$ & $\begin{array}{l}\text { "To rapidly review the existing } \\
\text { qualitative literature to identi- } \\
\text { fy perspectives from the com- } \\
\text { munity and drug distributors. } \\
\text { We focused on factors influ- } \\
\text { encing feasibility of planning }\end{array}$ & $\begin{array}{l}\text { Qualita- } \\
\text { tive }\end{array}$ & • 2002 to 2017 \\
and carrying out campaigns & - Global \\
and acceptability of MDA with- & - Qualitative studies and qualitative data that can be ex- \\
in community settings" & tracted from mixed-methods studies \\
& $\begin{array}{l}\text { - Studies that "discussed community and/or drug distribu- } \\
\text { tor perceptions of and experiences with any form of MDA } \\
\end{array}$ & $\begin{array}{l}\text { for LF elimination. Community encompasses people re- } \\
\text { ceiving treatment as well as those around them. A drug } \\
\text { distributor can be anyone distributing medicines" }\end{array}$
\end{tabular}

Abbreviations: MDA: mass drug administration.

Table 3. MEDLINE search strategy

\begin{tabular}{ll}
\hline Search & Query \\
\hline$\# 1$ & Search filaria* Field: Title/Abstract \\
\hline$\# 2$ & Search "Elephantiasis, Filarial"[Mesh] \\
\hline$\# 3$ & Search "Filariasis"[Mesh] \\
\hline$\# 4$ & Search lymphedema Field: Title/Abstract \\
\hline$\# 5$ & Search lymphoedema Field: Title/Abstract \\
\hline$\# 7$ & Search "Wuchereria bancrofti"[Mesh] OR "Brugia malayi"[Mesh] \\
\hline
\end{tabular}

Mass drug administration for filariasis: community views and programme design influences - a qualitative evidence synthesis (Protocol) 
Table 3. MEDLINE search strategy (Continued)

\begin{tabular}{|c|c|}
\hline \#8 & Search mass drug administration Field: Title/Abstract \\
\hline \#9 & Search "Mass Drug Administration"[Mesh] \\
\hline$\# 10$ & Search "mass administration" Field: Title/Abstract \\
\hline$\# 11$ & Search "coordinated administration" Field: Title/Abstract \\
\hline \#12 & Search "mass treatment " Field: Title/Abstract \\
\hline$\# 13$ & Search "mass distribution" Field: Title/Abstract \\
\hline \#14 & Search "coordinated distribution" Field: Title/Abstract \\
\hline$\# 15$ & Search $((((((\# 14)$ OR \#13) OR \#12) OR \#11) OR \#10) OR 9) OR \#8 \\
\hline \#16 & Search (\#15) AND \#7 \\
\hline$\# 17$ & Search "Qualitative Research"[Mesh] \\
\hline \#18 & $\begin{array}{l}\text { Search "focus group" or "grounded theory" or "narrative analys*" or "lived experience*" or "life } \\
\text { experience*" or "theoretical sampl*" or purposive Field: Title/Abstract }\end{array}$ \\
\hline \#19 & $\begin{array}{l}\text { Search semi-structured OR semistructured OR "structured categor*" OR "unstructured categor*" } \\
\text { OR "action research" OR (audiorecord* OR tape recorded *or videorecord* OR videotap } \text { OR }^{\star} \text { (au- } \\
\text { dio OR tape OR video*) OR interview* OR quasi-experiment* OR "case stud }{ }^{\star} \text { Field: Title/Abstract }\end{array}$ \\
\hline \#20 & Search "Interviews as Topic"[Mesh] OR "Interview" [Publication Type] \\
\hline \#21 & Search "Focus Groups"[Mesh] \\
\hline \#22 & $\begin{array}{l}\text { Search qualitative or ethno* or emic or etic or phenomenology* or hermeneutic* Field: Title/Ab- } \\
\text { stract }\end{array}$ \\
\hline \#21 & Search "Surveys and Questionnaires"[Mesh] \\
\hline \#22 & Search "Self Report"[Mesh] \\
\hline \#23 & Search "Anthropology, Cultural"[Mesh] \\
\hline \#24 & $\begin{array}{l}\text { Search collaborat }{ }^{\star} \text { or consultat }{ }^{\star} \text { or experience or involve }{ }^{\star} \text { or narrative* or opinion* } \text { or participat }^{\star} \\
\text { or partner }^{\star} \text { or perspective } \text { or story or stories Field: Title/Abstract }^{\star}\end{array}$ \\
\hline \#25 & Search $(((((()((\# 24)$ OR \#23) OR \#22) OR \#21) OR \#20) OR \#19) OR \#18) OR \#17 \\
\hline \#26 & Search (\#25) AND \#16 Filters: Publication date from 2000/01/01 \\
\hline
\end{tabular}

This is the preliminary search strategy for MEDLINE (PubMed). It will be adapted for other electronic databases. All search strategies will be reported in full in the final version of the review.

\section{APPENDICES}

\section{Appendix 1. EPPI Centre quality assessment tool}


1. Were steps taken to increase rigour in the sampling? Consider whether: *the sampling strategy was appropriate to the questions posed in the study (e.g. was the strategy well-reasoned and justified?); *attempts were made to obtain a diverse sample of the population in question (think about who might have been excluded; who may have had a different perspective to offer); ${ }^{*}$ characteristics of the sample critical to the understanding of the study context and findings were presented (i.e. do we know who the participants were in terms of, for example, basic socio-demographics, characteristics relevant to the context of the study, etc.)
Yes, a fairly thorough attempt was made.

Yes, several steps were taken. Yes, a few steps were taken. No, not at all/not stated/can't tell

Yes, a fairly thorough attempt was made.

Yes, several steps were taken. Yes, minimal/few steps were taken.

No, not at all/not stated/can't tell used?); ${ }^{*}$ steps were taken to ensure that all participants were able and willing to contribute (e.g. processes for consent, language barriers, power relations between adults and children/young people)

3. Were steps taken to increase rigour in the analysis of the data? Consider whether: *data analysis methods were systematic (e.g. was a method described/can a method be discerned?); *diversity in perspective was explored; * (if qualitative) the analysis was balanced in the extent to which it was guided by preconceptions or by the data; *the analysis sought to rule out alternative explanations for findings (in qualitative research, this could be done by, for example, searching for negative cases/exceptions, feeding back preliminary results to participants, asking a colleague to review the data, or reflexivity; in quantitative research, this may be done by, for example, significance testing)

4. Were the findings of the study grounded in/supported by the data? Consider whether: *enough data are presented to show how the authors arrived at their findings; *the data presented fit the interpretation/support claims about patterns in data; ${ }^{\star}$ the data presented illuminate/illustrate the findings; * (for qualitative studies) quotes are numbered or otherwise identified and the reader can see that they don't just come from one or two people

5. Please rate the findings of the study in terms of their breadth and depth. Consider whether (NB: it may be helpful to consider 'breadth' as the extent of description and 'depth' as the extent to which data have been transformed/analysed); *a range of issues are covered; *the perspectives of participants are fully explored in terms of breadth (contrast of two or more perspectives) and depth (insight into a single perspective); *richness and complexity have been portrayed (e.g. variation explained, meanings illuminated); ${ }^{*}$ there has been theoretical/conceptual development

6. To what extent does the study privilege the perspectives and experiences of children? Consider * whether there was a balance between open-ended and fixed response options; * whether children were involved in designing the research; * whether there was a balance between the use of an a priori coding framework and induction in the analysis; *the position of the researchers (did they consider it important to listen to the perspectives of children?); * whether steps were taken to assure confidentiality and put young people at ease

\section{Overall, what weight would you assign to this study in terms of the reliability/trustworthiness of} its findings? Guidance: think (mainly) about the answers you have given to questions 1 to 4 above

8. What weight would you assign to this study in terms of the usefulness of its findings for this review? Guidance: think (mainly) about the answers you have given to questions 5 and 6 above, and consider *the match between the study aims and findings and the aims and purpose of the synthesis; *its conceptual depth/explanatory power
Yes, a fairly thorough attempt was made.

Yes, several steps were taken. Yes, minimal/few steps were taken.

No, not at all/not stated/can't tell

Good grounding/support.

Fair grounding/support. Limited grounding/support
Limited breadth or depth. Good/fair breadth but very little depth.

Good/fair depth but very little breadth.

Good/fair breadth and depth

Not at all

A little

Somewhat

A lot

Low

Medium

High

Low

Medium

High

\section{H I S T O R Y}

Protocol first published: Issue 6, 2020

Mass drug administration for filariasis: community views and programme design influences - a qualitative evidence synthesis (Protocol) 


\section{CONTRIBUTIONS OF AUTHORS}

Melissa Taylor (MT) developed and revised the protocol.

Paul Garner (PG) commented on the protocol and contributed to the background and methodology.

Sandy Oliver (SO) commented on the protocol and contributed to the methodology.

All authors approved the final protocol version.

\section{DECLARATIONS OF INTEREST}

Melissa Taylor and Paul Garner are employed by the Liverpool School of Tropical Medicine (LSTM). LSTM is a world leader in promoting mass drug administration (MDA) for filariasis through its previous Director, Professor David Molyneux. LSTM continues to receive grants from a variety of sources to help implement MDA programmes in filariasis, including COUNTDOWN, which is committed to development of trials and mass treatment programmes related to NTDs, valued at GBP 7 million from 2014 onwards. From 2014 to 2017 , Paul Garner received salary support from the COUNTDOWN Research Consortium.

Sandy Oliver has no known conflicts of interest.

\section{SOURCES OF SUPPORT}

\section{Internal sources}

- Liverpool School of Tropical Medicine, UK

\section{External sources}

- Department for International Development, UK

Project number 300342-104 